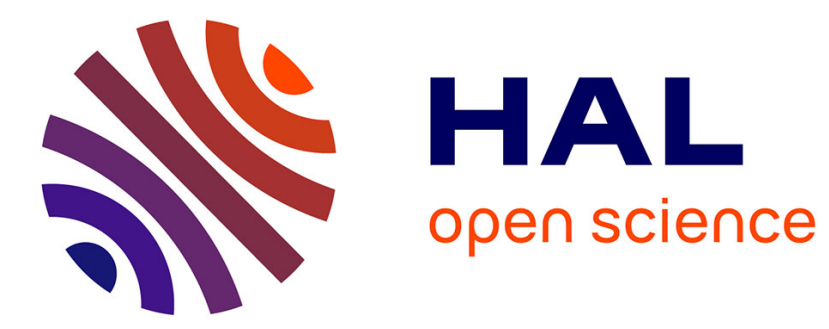

\title{
Peaks, Slumps, and Bumps: Individual Differences in the Development of Creativity in Children and Adolescents
}

\author{
Baptiste Barbot, Todd I Lubart, Maud Besançon
}

\section{To cite this version:}

Baptiste Barbot, Todd I Lubart, Maud Besançon. Peaks, Slumps, and Bumps: Individual Differences in the Development of Creativity in Children and Adolescents. New Directions for Child and Adolescent Development, 2016, 2016, pp.33 - 45. 10.1002/cad.20152 . hal-01392496

\section{HAL Id: hal-01392496 \\ https://hal.parisnanterre.fr/hal-01392496}

Submitted on 9 Nov 2016

HAL is a multi-disciplinary open access archive for the deposit and dissemination of scientific research documents, whether they are published or not. The documents may come from teaching and research institutions in France or abroad, or from public or private research centers.
L'archive ouverte pluridisciplinaire $\mathbf{H A L}$, est destinée au dépôt et à la diffusion de documents scientifiques de niveau recherche, publiés ou non, émanant des établissements d'enseignement et de recherche français ou étrangers, des laboratoires publics ou privés. 
RUNNING HEAD: PEAKS, SLUMPS AND BUMPS

Peaks, Slumps and Bumps: Individual differences in the Development of Creativity in Children and Adolescents

Baptiste Barbot, Todd I. Lubart, Maud Besançon

\begin{abstract}
This article reviews developmental studies of creativity in children and adolescents with a focus on "peaks" and "slumps" that have often been described in the literature. The irregularity of creativity development is interpreted in light of conceptual and measurement issues, and with regard to the interaction between individual-level resources, task-specific demands, and environmental influences resulting in apparent individual differences in the development of creativity. The need for longitudinal designs, multi-dimensional, and multidomain assessment of creative potential limiting the contribution of task-specific factors, is outlined and discussed as an important direction for developmental research on creativity.
\end{abstract}

Keywords: Fourth-grade Slump, Creativity Development, Measurement, Domainspecificity 
PEAKS, SLUMPS AND BUMPS

Peaks, Slumps and Bumps: Individual differences in the Development of Creativity in Children and Adolescents

"Peaks", "slumps" and "bumps": there are not enough terms to describe the discontinuityin the development of creativity throughout the lifespan. Illustrated by the livesof eminent creators, " $a$ work that brings its creator unprecedented acclaim may be followed by an embarrassing fiasco" (Simonton, 2000; pp. 312). What are the common developmental trends in the development of creativity and why are there differences in these trends across cultures, school environments, and people? Why do some children experience a creativity slump and others do not? Why do some children never "recover" from a creativity slump?

After presenting a general framework on creative potential and conceptual distinctions with creative achievement, this article addresses these general questions by reviewing classic and recent developmental studies of creativity in children and adolescents, with a focus on patterns of discontinuity that have been observedin the literature. We outline multiple explanatory causes of these patterns and discuss methodological issues of studies showing these trends. Finally, we present a model of creativity development that explains individual differences in creativity outcomes in light of the interaction between individual-level resources, environmental influences, and task-specific demands. We conclude by discussing important areas of focus for the field's research agenda.

\section{Nature of Creativity and Creative Potential}

Creativity is the ability to produce original work that fits within particular task or domain constraints (e.g., Sternberg \& Lubart, 1995). Consistent with this classic definition, which emphasizes the conceptof "an ability" (rather than a set of "abilities"), it is often thought that creativity represents a generalized or unitary entity ("g-factor view"), despites 
PEAKS, SLUMPS AND BUMPS

evidences for its multidimensionality and domain-specificity reported since decades (e.g., Baer, 1998; Wallach, 1970). This g-factor view, however, leads to misconceptionsabout the nature of creativity and its development (Barbot, Besançon, \& Lubart, 2015). In this regard, it is important to distinguish first between creative achievement and creative potential, as each couldfollow fairly distinct developmental pathways.

Creative achievement refers to an accomplished creative output, recognized as valuable in a domain-based context. This output may be seen on a unidimensional continuum ranging from low to outstanding creativity depending on its level of originality and appropriateness. Although a creative output can be evaluated on such aunidimensional continuum, this does not indicate that it results from a single ability (Barbot \& Tinio, 2015). Componential approaches have posited that creativity results from people's unique combination of multiple resources coming into play in creative work, including aspects of cognition, motivation, and personality (e.g., Lubart, 1999; Sternberg \& Lubart, 1995).

Extending this view, we have recently posited that this set of person-level resources interacts with each particular creative task's characteristics and demands and that, ultimately, a person's creative potential will depend on the "quality of fit" between her unique profile of resources and the demands of a given creative task (e.g., Barbot \& Tinio, 2015; Barbot et al., 2015; Lubart, Zenasni, \& Barbot, 2013). Consistent with extant "hybrid" models of creativity (e.g., Plucker \& Beghetto, 2004; Kaufman \& Baer, 2004), this view suggests that there are as many potentials for creativity as there are creative tasks (i.e., outlets in which outcomes can be evaluated on the creativity continuum). With the same unique profile of resources, a person can show a high potential for poetry writing, average potential for fiction writing, and low potential for musical composition. This heterogeneity is expected because these creative outlets rely conceptually on a different mixture of person-level resources. Because a given creative task may rely on a very specific combination of resources, the likelihood that a 
PEAKS, SLUMPS AND BUMPS

person's profile of resources optimally fitsthat task's requirement is very low. As a result, the probability of achieving an outstanding level of creativity in that task is very low and most outcomes will be of average or low creativity (depending on how "misfitting" the person's profile of resources is to the task's requirements). Furthermore, it is likely that a person's creative potential will never lead to creative achievement in a given task (regardless of the quality of fit between her potential and that task) if she doesn't have the opportunity to engage in that task, or doesn't choose to invest time and energy in that task (e.g., Amabile, 1996).

This "optimal-fit view" helps understand the rarity of exceptional creativity outcomes (big-C creativity) while shedding light onto the issue of domain generalityspecificity of creativity (i.e., it is not likely that multiple creative tasks across domains will require the same "optimal" combination of resources). It should, however, not be strictly interpreted as if each person's profile of resources is static, or could be compared to a "winning bingo ticket" when interacting with the right task. Individual inclinations, interests, and personal investments remain at the center of creative work, and are deeply and continuously shaped by environmental influences and experiences. These influences (which can be seen as part of the person's potential for creativity) may at least partly explain patterns of discontinuity of creativity throughout the lifespan.

\section{Discontinuity of the Development of Creative Potential}

Because creativity is multifaceted and partly domain- (and task-) specific, creative potential doesn't develop as a monolithic entity: it is multidimensional and each task-specific potential results from the development of the multiple contributing person-level resources. Among them, divergent thinking (DT) is by far the most widely studied froma developmental perspective. DT is the ability to produce multiple solutions or ideas in response to a single problem or stimulus, such as generating alternative uses for a common object. Recent research has focused on DT of pre-school children as young as two years old (Bijvoet-van \& Hoicka, 
PEAKS, SLUMPS AND BUMPS

2014) and concluded that individual differences in DT start emerging during pre-school age, and then, that DT performance tends to increase. This general trend seems to continue until about age 40, followed by "systematic maturational declines" (McCrae, Arenberg, \& Costa, 1987; pp.136), and is punctuated by multiple patterns of discontinuity especially during childhood and adolescence.

Based on several longitudinal and cross-cultural studies,Torrance (1967) produced a comprehensive report on the issue of DT development and discontinuity from childhood to early adolescence. The general trend described by Torrance, confirmed by several subsequent studies, can be summarized by an overall increase of DT from pre-school age to early adolescence with three common "slumps" (steep and temporary decrease in DT performance). The first slump occursaround age 5, the second around age 9 (fourth-grade slump) and the third around age 12. These slumps, and in particular the "fourth-grade" slump, have received substantial attention despites issues of replicability, cross-cultural differences, and considerable individual differences regarding the existence, timing and domain in which a slump is or is not observed.

As an illustration, Charles and Runco (2001) identified a significant peak, rather than slump, in the divergent thinking of fourth-grade children. Lau and Cheung (2010) did notidentifya fourth grade slumpof Chinese students, but rather, sixth- and seventh-grade slumps, with marked gender differences in developmental patterns of DT. Similarly, Besançon and Lubart (2008)demonstrated a different timing of the slumps of French children in middle-school, that seemed to vary according to the pedagogical context and the nature of the task administered. Claxton, Pannells, and Rhoads(2005) identified a decrease of the cognitive aspects of creativity at the onset of puberty, similar to $\mathrm{Yi}, \mathrm{Hu}$, Plucker, and McWilliams (2013) showing a general decline of DT of Chinese students between 12 to 14 years old, followed by an increase of small magnitude. In contrast, Kleibeuker, De Dreu,and 
PEAKS, SLUMPS AND BUMPS

Crone(2013) showed a relative peak of visuo-spatial DT around 15 years old, but no agerelated difference in verbal DT.

Other person-level resources that contribute to creative potential have been investigated and, similar to developmental patterns of DT, have often been described by nonlinear trajectories. In adolescence, insight ability seems to increase with age (Kleibeuker et al., 2013), as does the accuracy of original ideas selection and the preference for appropriate ideas (Charles \& Runco, 2001). Claxton and colleagues (2005) have suggested a peak of "divergent feeling" (including curiosity, complexity, or risk-taking) around $9^{\text {th }}$ gradethat appears in the same wave asa slump in DT. These patterns are consistent with research showing how motivational dimensions and personality trait development contribute to variability in the developmental trajectory of creative potential in various domains (Hong, Peng, \& O'Neil Jr., 2014). Personality development could, in addition, follow different developmental pathways according to gender, reflecting differences in the timing of the psychosocial stages experienced by girls and boys. For example, openness to experience related to creative potential in music, visual arts, and creative writing (but less to science and technology; Hong et al., 2014) showsan increasing trend in adolescence girls and a decreasing onein adolescent boys (e.g., Branje, van Lieshout, \& Gerris, 2007). Internalized age-related stereotypes (and perhaps gender-related stereotypes), and age-related differentiation in beliefs about personality development could also impact the expression of personality features related to creativity (Hui et al., 2014). Hence, it is expected that boys and girls differ in their creative potential development in various creative outlets based upon multipleconative variables that follow distinct developmental trajectories.

As a last example, domain-specific expertise and domain-based knowledge, crucial for creative achievement, can also be described by non-monotonic longitudinal trends. Based on a hisotriometric analysis of 59 classical composers, Simonton (2000) suggests that complex 
PEAKS, SLUMPS AND BUMPS

specialization and versatility effects determine creative development across the life span of eminent creative persons. This "Creative-expertise" hypothesis, could easily apply to everyday life creativity, as found in children and adolescents, given the necessary contribution of domain-based knowledge in any creative work.

\section{Foundations of Individual Differences in Creativity Development}

As outlined above, there is discontinuity in the development of creativity during childhood and adolescencewith "slumps" frequently cited, but when they occur in a study sample, there is variability as to whether or not a given child will experience the slump, when it is experienced, in which type of creative outlet the slump is experienced, and the outcome of the slump (some children might not "recover" after experiencing a slump; Charles \& Runco, 2001; Runco, 2014). These differences can be explained by three sets of complementary explanations: (1) Asynchronicity (asynchronous development of individual resources involved in creative potential), (2) Environmental influences, and (3) Taskspecificity (creative task characteristics and other methodological artifacts).

- Asynchronicity. Patterns of discontinuity can be understood in light of the "natural", and partly genetically grounded development of the various resources that contribute to creative potential (Barbot, Tan, \& Grigorenko, 2013). Using a "stage" approach, Piaget suggested that children through fourth grade are developmentally "concrete thinkers" which aligns with the idea of the fourth-grade slump, as children remain consistent with reality and fail to propose ideas that are more original. Approaches that have relied on "wave of development" have suggested that all components of the mind are not developing at the same time, and that the weakening of creative thinking could be related to the emergence of new skills in other areas. In this line, Lubart and Lautrey (1996) showed a fourth-grade slump in DT associated with a substantial increase in logical reasoning. They interpreted this result as a compensating effect, in which the "wave" of DT might be in a "slump"when the wave of 
PEAKS, SLUMPS AND BUMPS

reasoning is at its "peak". Many similar examples can be found in the neurodevelopmental literature, highlighting, for example, a temporal gap between the development of the socioemotional system and the cognitive control systems in adolescence, leading to maladaptive behavior such as risk taking and impulsivity (e.g., Barbot \& Hunter, 2012), which could also have implication in the development of person-level resources important for creativity.

- Environmental influences. According to Torrance (1968) creativity slumps can be explained by normative effects of the school environment, as they happen at times when children are taught to respect school rules and fit within the classroom norms. This explanation finds support in research showing slumps at different points in time depending on the specific contextual and cultural demands. For example, Yi and colleagues (2013) suggest that the emphasis on college entrance exams taken during high-school in China raises expectations for academic performance in middle-school, explaining cultural differences in DT trajectories (pressures have moved into earlier grades in this culture). These effects can be moderated by teacher's influences (Davies et al., 2014) and the type of pedagogy encouraged (Besançon \& Lubart, 2008; Besançon, Lubart, \& Barbot, 2013).

The family environment is also a critical determinant of creative potential development. Here too, the cultural context has an indirect influence through common parental practices that are prevalent in a culture (Mourgues, Barbot, Tan, \& Grigorenko, 2014). For example, Fearon, Copeland, and Saxon (2013) noted the negative impact of authoritarian parenting on the development of creativity of Jamaican youth, a common parenting style in this culture. Consistent with prior research on parenting and creativity, this result is in line with the idea that creativity flourishes when developmental experiences and conditions are optimal (Dai et al., 2012; Runco \& Cayirdag, 2013). 
PEAKS, SLUMPS AND BUMPS

Recent research, however, tends to challenge this assumption. For example, Dahlman, Bäckström, Bohlin and Frans (2013) show that street children in Bolivia were far more efficient in specific DT tasks (generating alternative uses of a common object) compared to children of low SES living with their parents. They concluded that street children gained "training" in this type of tasks given the nature of their daily-life experience (perhaps requiring some of the skills engaged by alternative uses tasks). Similarly, Damian and Simonton (2015) make the hypothesis that experiences of developmental adversity (such as early parental death, poverty, or psychopathology) might foster unconventional ideas. Based on an analysis of eminent African Americans they concluded that "diversifying experiences" push people outside the realm of "normality", and in turn, influence the development of creativity.

Together, this body of work supports that both individual and environmental influences jointly contribute to creativity development (Niu, 2007). Because the influence of the school and family environment is not systematic and because the status of adversity as a condition that impedes creativity development is unclear, environment-level resources should be further explored and considered in their interaction with person-level resources (e.g., "absorption level" of environmental influences), as well as with tasks requirements.

- Task-specificity and methodological artifacts. Several methodological limitations and artifacts contribute to the apparent discontinuity of creativity development. First, developmental studies of creativity often suffer from small sample sizes, selection biases (alternative tracks underrepresented), and the use of cross-sectional design (focusing on agegroup differences) making it difficult to untangle cohort effects from actual developmental effects. Even longitudinally, classroom and teachers' effects outlined above could bias developmental inferences (it is not likely that participants will "benefit" equally from these effects from grade to grade). 
PEAKS, SLUMPS AND BUMPS

Most importantly, the different nature and emphasis of tasks used in studies may explain variations in the existence and timing of slumps (Charles \& Runco, 2001): almost all studies that have administered multiple tasks to the same participants have outlined distinct developmental trajectories for each task (Besançon \& Lubart, 2008; Claxton et al., 2005; Kleibeuker et al., 2013; Torrance, 1968) confirming that not all tasks are equivalent when it comes to measure creative potential (specific demands varies as a function of the task, even within the same domain of production; Wu, Cheng, Ip, \& McBride-Chang, 2005).

Relatedly, one of the most challenging issues in longitudinal studies of creativity is the reliance on similar but non-paralleltasks at different measurement occasions. Because production-based tasks require producing original and unique outputs, administering strictly the same task at multiple measurement occasions makes little sense: "memory" effects, carryover effects, and other "learning" biases may lead to rote production of outputs already proposed by the child or traded with a classmate at earlier testing sessions. However, by exposing children to a slightly different task at each measurement occasion (e.g., alternative uses for a brick vs. a cardboard box), it becomes challenging to isolate a person's “true change" in the tested ability as distinct from change related to variations in the task's characteristics. Indeed, these characteristics don't have a systematic effect: at a given time, one may be more "inspired" when providing alternative uses for a brick rather than for a cardboard box. This is highlighted by the large proportion of task-specific variance in creative potential tests scores as suggested by low to moderate inter-task correlations (Barbot, Besançon,\& Lubart, Submitted; Dumas \& Dunbar, 2014).

\section{Towards an Optimal-fit view ofCreative Potential in Development}

As outlined by Simonton (2000), “at any given time, the performance outcome for a particular work in a specified form or genre will be contingent on a chaotic mixture of influences, some beneficial and others deleterious" (pp. 313). This mechanism is likely to 
PEAKS, SLUMPS AND BUMPS

occur at every level of the creativity continuum, including in "everyday" accomplishments of children and adolescents. Specifically, we have posited earlier that individual differences in creativity outcomes result from the interaction between individual-level resources and taskspecific demands. This "optimal-fit" view translates easily in a developmental perspective: performance outcomes in a given creative outlet will depend upon the specific creative-task characteristics and the (asynchronous) development of person-level characteristics (e.g., DT and other cognitive factors, personality, motivational and emotional characteristics). At different points in time, the resulting multivariate profile of person-level resources will be more or less in linewith the particular task requirements, leading to outcomes of variable creativity over time. This idea is illustrated in Figure 1.

As showed in Figure 1a, the various person-level resources of a child develop asynchronously from childhood to adolescence following distinct trajectories (for the sake of clarity, only the hypothetical development of selected resourcesis represented). Figure 1b, represents two tasks' characteristics in terms of required level of these person-level resources. Finally, Figure 1c illustrates the "quality of fit" between the children's multivariate profile of person-level resources at different point in time and tasks A and B requirements. This quality of fit represents the child's creative potential for each "target" task (Figure 1b). As represented, the hypothetical child may have a higher creative potential for task B than for task A at 10 years old: the quality of fit between the child's profile of resources and Task B's demands is better than for Task A's demands. However, at 12 years old, the quality of fit has diminished due to the "natural" development of the child's profile of resources. This profile is now more aligned with Task A's demands, than with Task B.

Environmental influences are not incorporated in this illustration but they could be represented as forces that steer each person-level resource in various directions, depending on their interaction with these resources. Hence, environmental influences have an indirect effect 
PEAKS, SLUMPS AND BUMPS

on creative potential in a given task (Figure 1c) through their beneficial (or harmful) influence on each person-level resource.

\section{Conclusion}

In this article, we have outlined an "optimal-fit" view of creativity according to which the creativity of a product depends on the fit between a creative task characteristic and a person's profile of resources underlying creativity in that task. By extension, the apparent discontinuity of creativity development in childhood and adolescence can be understood in light of (1) asynchronous development of person-level resources contributing to creativity, (2) environmental influences (orienting the development of person-level resources), and (3) task characteristics.

We have also outlined a number of conceptual and methodological limitations to the study of creativity development, and, in particular, the need to capture its multidimensional and partly domain-specific nature as it develops: creativity development is not a generalized process, as creativity is not equivalent to the sum of its constituting "ingredients" and certainly not equivalent to a single ingredient, such as DT. Additionally, we have pointed out the critical need for longitudinal evidence rather than age-group comparisons in the study of creativity development.

These endeavors may, however, prove challenging. Indeed, "because of the multifaceted nature of creativity, there has been considerable discussion concerning how to measure creativity as it develops" (Claxton et al., 2005; pp. 328). This discussion has fallen short these past few years, as challenges specific to the study of creativity development are surprisingly disregarded in developmental investigations (e.g., issues of task-specificity and repeated measurements, untangling cohorts and classroom effects from true change). Therefore, it follows that important directions for the field are(1) the development of new measures specially designedforlongitudinal studies (e.g., limiting the contribution of task- 
specific factors), or devising new scoring methods for existing instrument that would reduce common biases when used longitudinally, and/or (2), the development of methodological and statistical paradigms that would control for high task-specificity and other methodological biases impeding the study of "true change" in creativity research.

\section{References}

Amabile, T. M. (1996). Creativity in context. Boulder, CO: Westview.

Baer, J. (1998). The case for domain specificity in creativity. Creativity Research Journal, 11, 173-177.

Barbot, B., \& Hunter, S. R. (2012). Developmental changes in adolescence and risks for delinquency. In E. L. Grigorenko (Ed.), Handbook of juvenile forensic psychology and psychiatry (pp. 11-34). New York, New York: Springer. doi:10.1007/978-1-4614-0905-2

Barbot, B., Tan, M., \& Grigorenko, E. L. (2013). The genetics of creativity: The generative and receptive sides of the creativity equation. In O. Vartanian, A. Bristol \& J. C. Kaufman (Eds.), The neuroscience of creativity (pp. 71-93) MIT Press.

Barbot, B., Besançon, M., \& Lubart, T. (2015). Creative potential in educational settings: Its nature, measure, and nurture. Education 3-13, 43(4), 371-381. doi:10.1080/03004279.2015.1020643

Barbot, B., Besançon, M., \& Lubart, T. (Submitted). The generality-specificity of creativity: Exploring the structure of creative potential with EPoC.

Barbot, B., \& Tinio, P. P. L. (2015). Where is the "g" in "creativity"•? a specializationdifferentiation hypothesis. Frontiers in Human Neuroscience, 8(1041) doi:10.3389/fnhum.2014.01041

Besançon, M., \& Lubart, T. I. (2008). Differences in the development of creative competencies in children schooled in diverse learning environments. Learning and Individual Differences, 18(4), 381-389.

Besançon, M., Lubart, T. I., \& Barbot, B. (2013). Creative giftedness and educational opportunities. Educational and Child Psychology., 30(2), 79-88.

Bijvoet-van, d. B., \& Hoicka, E. (2014). Individual differences and age-related changes in divergent thinking in toddlers and preschoolers. Developmental Psychology, 50(6), 16291639. doi:10.1037/a0036131

Branje, S. J. T., van Lieshout, C. F. M., \& Gerris, J. R. M. (2007). Big five personality development in adolescence and adulthood. European Journal of Personality, 21(1), 4562.

Charles, R. E., \& Runco, M. A. (2001). Developmental trends in the evaluative and divergent thinking of children. Creativity Research Journal, 13(3), 417-437.

Claxton, A. F., Pannells, T. C., \& Rhoads, P. A. (2005). Developmental trends in the creativity of school-age children. Creativity Research Journal, 17(4), 327-335. doi:10.1207/s15326934crj1704_4 
Dahlman, S., Bäckström, P., Bohlin, G., \& Frans, Ö. (2013). Cognitive abilities of street children: Low-SES bolivian boys with and without experience of living in the street. Child Neuropsychology, 19(5), 540-556.

Dai, D. Y., Tan, X., Marathe, D., Valtcheva, A., Pruzek, R. M., \& Shen, J. (2012). Influences of social and educational environments on creativity during adolescence: Does SES matter? Creativity Research Journal, 24(2-3), 191-199.

Damian, R. I., \& Simonton, D. K. (2015). Psychopathology, adversity, and creativity: Diversifying experiences in the development of eminent african americans. Journal of Personality and Social Psychology, 108(4), 623-636. doi:10.1037/pspi0000011

Davies, D., Jindal-Snape, D., Digby, R., Howe, A., Collier, C., \& Hay, P. (2014). The roles and development needs of teachers to promote creativity: A systematic review of literature. Teaching and Teacher Education, 41, 34-41. doi:10.1016/j.tate.2014.03.003

Dumas, D., \& Dunbar, K. N. (2014). Understanding fluency and originality: A latent variable perspective. Thinking Skills and Creativity, 14, 56-67. doi:10.1016/j.tsc.2014.09.003

Fearon, D. D., Copeland, D., \& Saxon, T. F. (2013). The relationship between parenting styles and creativity in a sample of jamaican children. Creativity Research Journal, 25(1), 119128.

Hong, E., Peng, Y., \& O'Neil Jr., H. F. (2014). Activities and accomplishments in various domains: Relationships with creative personality and creative motivation in adolescence. Roeper Review: A Journal on Gifted Education, 36(2), 92-103. doi:10.1080/02783193.2014.884199

Hui, A. N., Yeung, D. Y., Sue-Chan, C., Chan, K., Hui, D. C., \& Cheng, S. (2014). Gains and losses in creative personality as perceived by adults across the life span. Translational Issues in Psychological Science, 1(S), 28-34. doi:10.1037/2332-2136.1.S.28

Kaufman, J. C., \& Baer, J. (2004). The Amusement Park Theoretical (APT) Model of creativity. Korean Journal of Thinking and Problem Solving, 14, 15-25.

Kleibeuker, S. W., De Dreu, C. K., \& Crone, E. A. (2013). The development of creative cognition across adolescence: Distinct trajectories for insight and divergent thinking. Developmental Science, 16(1), 2-12.

Lau, S., \& Cheung, P. C. (2010). Developmental trends of creativity: What twists of turn do boys and girls take at different grades? Creativity Research Journal, 22(3), 329-336.

Lubart, T. I., \& Lautrey, J. (1996). Development of creativity in 9- to 10-year old children. . The Growing Mind Congress, Geneva, Switzerland.

Lubart, T. I. (1999). Componential models of creativity. In M. A. Runco, \& S. Pritzer (Eds.), (pp. 295-300). New York:NY: Academic Press.

Lubart, T. I., Zenasni, F., \& Barbot, B. (2013). Creative potential and its measurement. International Journal of Talent Development and Creativity, 1(2), 41-51.

McCrae, R. R., Arenberg, D., \& Costa, P. T. (1987). Declines in divergent thinking with age: Cross-sectional, longitudinal, and cross-sequential analyses. Psychology and Aging, 2(2), 130-137. doi:10.1037/0882-7974.2.2.130

Mourgues, C., Barbot, B., Tan, M., \& Grigorenko, E. L. (2014). The interaction between culture and the development of creativity. In L. Arnett Jensen (Ed.), The oxford handbook of human development and culture: An interdisciplinary perspective () doi:10.1093/oxfordhb/9780199948550.013.16 
Niu, W. (2007). Individual and environmental influences on chinese student creativity. The Journal of Creative Behavior, 41(3), 151-175. doi:10.1002/j.2162-6057.2007.tb01286.x

Plucker, J. A., \& Beghetto, R. A. (2004). Why creativity is domain general, why it looks domain specific, and why the distinction doesn't matter. In R. J. Sternberg, E. L. Grigorenko, \& J. L. Singer (Eds.), Creativity: From potential to realization. (pp. 153168). Washington, DC: APA.

Runco, M. A., \& Cayirdag, N. (2013). The development of Children's creativity. In O. N. Saracho, \& B. Spodek (Eds.), Handbook of research on the education of young children (Third Edition ed., pp. 102-114). New York, NY: Routledge.

Runco, M. A. (2014). Creativity. theories and themes: Research, development, and practice (Second Edition ed.). Waltham,MA: Elsevier.

Simonton, D. K. (2000). Creative development as acquired expertise: Theoretical issues and an empirical test. Developmental Review, 20(2), 283-318.

Sternberg, R. J. (2000). Identifying and developing creative giftedness. Roeper Review, 23(2)

Sternberg, R. J., \& Lubart, T. I. (1995). Defying the crowd: Cultivating creativity in a culture of conformity. New York, NY: Free Press.

Torrance, E. P. (1967). Understanding the fourth grade slump in creative thinking .( No. Report No. BR-5-0508; CRP-994). Washington, DC: U.S. Office of Education.

Torrance, E. P. (1968). A longitudinal examination of the fourth grade slump in creativity. Gifted Child Quarterly, 12(4), 195.

Wallach, M. A. (1970). Creativity. In P. H. Mussen (Ed.), Carmichael's manual of child psychology (Vol. 1, pp. 1211-1272) New York: Wiley

Wu, C. H., Cheng, Y., Ip, H. M., \& McBride-Chang, C. (2005). Age differences in creativity: Task structure and knowledge base. Creativity Research Journal, 17(4), 321-326. doi:10.1207/s15326934crj1704_3

Yi, X., Hu, W., Plucker, J. A., \& McWilliams, J. (2013). Is there a developmental slump in creativity in china? the relationship between organizational climate and creativity development in chinese adolescents. The Journal of Creative Behavior, 47(1), 22-40. 
Figure 1.Illustration of the "optimal-fit" view of creativity development.

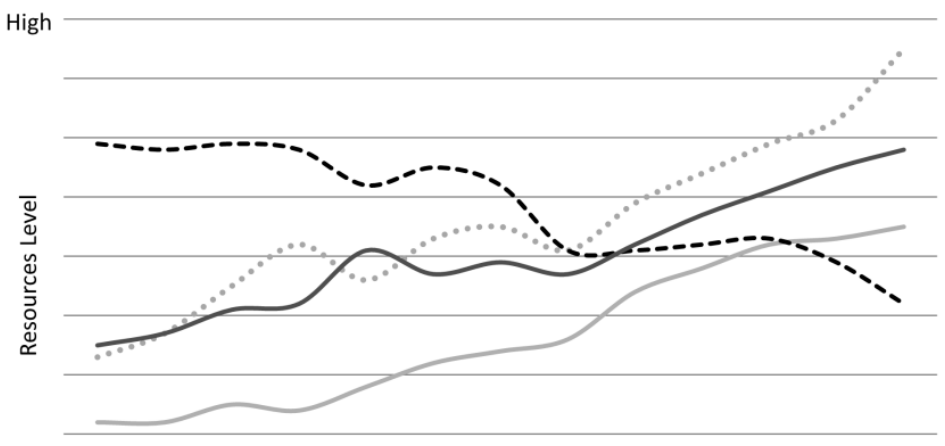

Low Age 5 Age 6 Age 7 Age 8 Age 9 Age 10 Age 11 Age 12 Age 13 Age 14 Age 15 Age 16 Age 17

(a) ..... Divergent Thinking Domain-based knowledge --notivation Openness

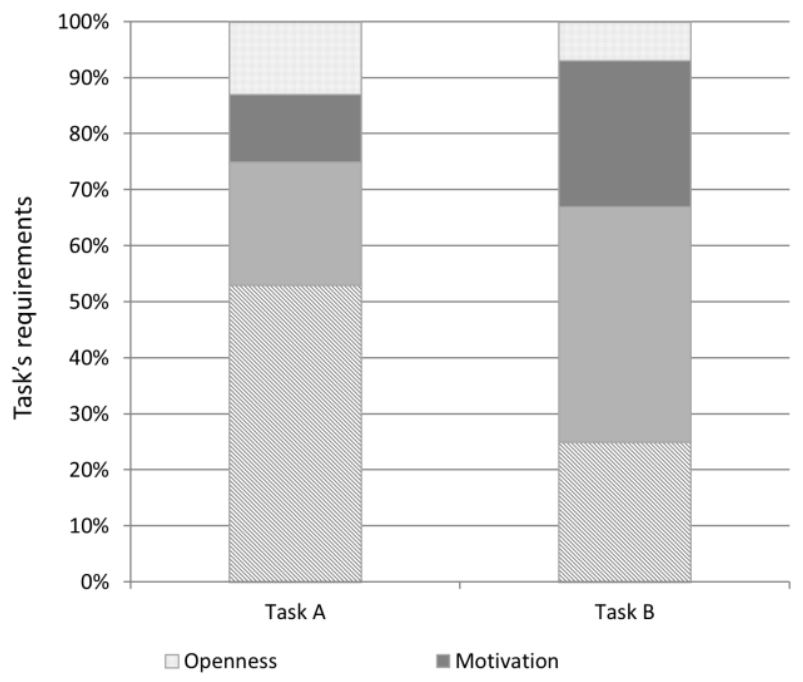

(b)

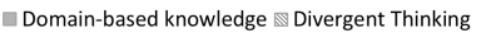

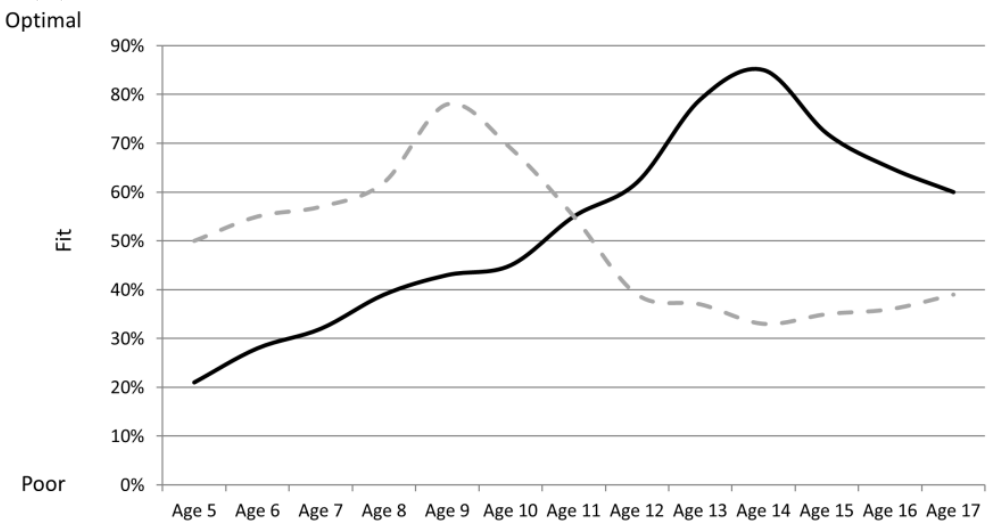

(c)

Note. Fig 1.a. Development of four person-level resources during childhood and Adolescence. Fig 1b. Two tasks characteristics in terms of person-level requirements. Fig 1c. "quality of fit" between the individual's multivariate profile of person-level resources (fig. 1a)and tasks characteristics (fig. 1b). 